TecnoLógicas

ISSN-p 0123-7799

ISSN-e 2256-5337

Vol. 22, No. 44

Enero-abril de 2019

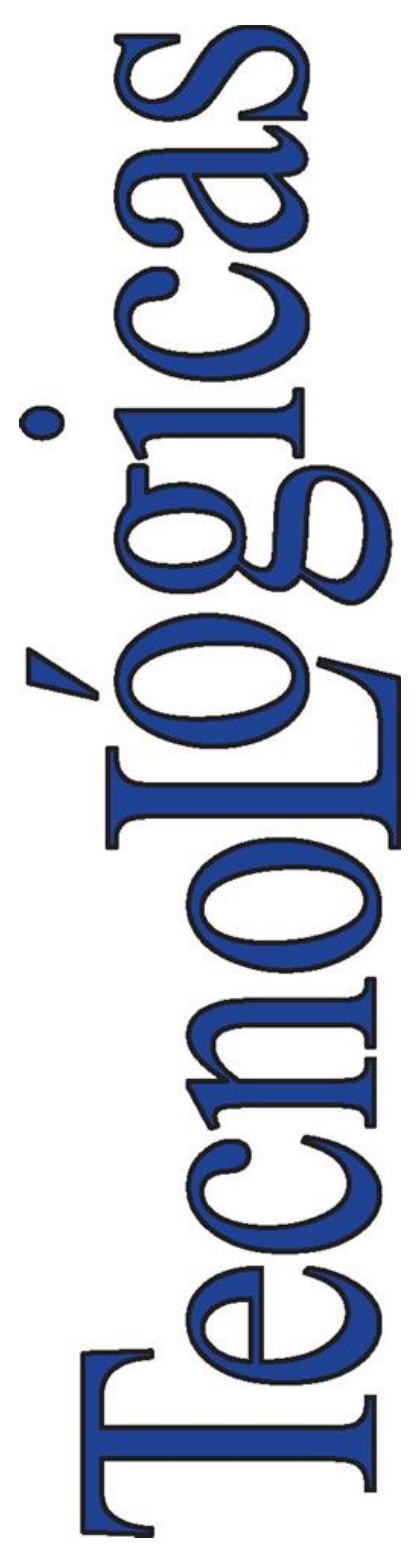

(C) Instituto Tecnológico Metropolitano Este trabajo está licenciado bajo una Licencia Internacional Creative Commons Atribución (CC BY-NC-SA)
Artículo de Investigación/Research Article

\title{
Caracterización magnética de material compuesto con matriz de resina epoxi y llanta en desuso reforzado con magnetita en diferentes proporciones
}

\section{Production and characterization of composite materials with epoxy resin matrices reinforced with tire rubber and magnetite in different proportions}

\author{
Felix Enrique Salinas Tacumá ${ }^{1}$, David Arsenio \\ Landinez Téllez ${ }^{2}$, Andrés Orlando Garzón Posada ${ }^{3}$ y \\ Jairo Roa Rojas ${ }^{4}$
}

Recibido: 05 de julio de 2018

Aceptado: 14 de diciembre de 2018

Cómo citar / How to cite

F. E. Salinas-Tacumá, D. A. Landinez-Téllez, A. O. Garzón-Posada y J. Rojas, Caracterización magnética de material compuesto con matriz de resina epoxi y llanta en desuso reforzado con magnetita en diferentes proporciones. TecnoLógicas, vol. 22, no. 44, pp. 81-95, 2019.

https://doi.org/10.22430/22565337.999

1 MSc. en Ciencias Física, Licenciado en Física, Grupo de Física de Nuevos Materiales, Departamento de Física, Universidad Nacional de Colombia, Bogotá-Colombia, flxud84@gmail.com

2 PhD en Física, MSc. en Física, Físico, Grupo de Física de Nuevos Materiales, Departamento de Física, Universidad Nacional de Colombia, BogotáColombia, dalandinezt@unal.edu.co

3 MSc. en Ingeniería: Materiales y Procesos de Manufactura, Ingeniero de Diseño y Automatización Electrónica, Grupo de Física de Nuevos Materiales, Departamento de Física, Universidad Nacional de Colombia, BogotáColombia, aogarzonp@unal.edu.co

4 PhD en Física, MSc. en Física, Físico, Grupo de Física de Nuevos Materiales, Departamento de Física, Universidad Nacional de Colombia, BogotáColombia,jroar@unal.edu.co 
Caracterización magnética de material compuesto con matriz de resina epoxi y llanta en desuso reforzado con magnetita en diferentes proporciones

\section{Resumen}

Este trabajo describe la producción y caracterización magnética de un compuesto a base de resina epoxi y caucho pulverizado reforzado con magnetita $\left(\mathrm{Fe}_{3} \mathrm{O}_{4}\right)$ en polvo. Además, se presenta el análisis realizado a los compuestos en función de la carga magnética dentro de la matriz polimérica. Las propiedades magnéticas del material se realizaron a diferentes temperaturas en un rango magnético de -30000 a 30000 0e. La morfología tanto de los precursores como de los compuestos fue determinada por microscopía electrónica de barrido (MEB) y la caracterización estructural fue realizada por difracción de rayos X (DRX). El patrón de difracción obtenido en las mediciones de DRX permite observar que, con la adición de magnetita en polvo en la matriz plástica, se crea una transición amorfo-cristalina en el material. A través de las imágenes obtenidas por MEB se observa la presencia de hematita en el precursor $\left(\mathrm{Fe}_{3} \mathrm{O}_{4}\right)$ y un tamaño irregular en las muestras. Los compuestos se comportan como un material magnético blando y presenta cambios en la variación de la fuerza coercitiva, tanto con el incremento de temperatura como con la variación de porcentaje de volumen de magnetita en la matriz polimérica. El material fabricado puede ser utilizado en aplicaciones donde el compuesto debe ser fácilmente detectable.

\section{Palabras clave}

Desechos de llantas, ferrimagnético, materiales compuestos, magnetita, resina epoxi.

\section{Abstract}

This work describes the production and magnetic characterization of a compound base on epoxy resin and powdered rubber reinforced with magnetite $\left(\mathrm{Fe}_{3} \mathrm{O}_{4}\right)$ poder. Also it is presented the análisis carried out on the components, which are according to the magnetic charging inside the polymeric matrix. the magnetic properties of the material they were made at different temperaturas in a magnetic range from -30000 to 30000 Oe the morphology of both the precursons like the compounds was determined by scanning electron microscopy and structural characterizatin was performed by X- ray diffraction (XRD). The diffraction pattern obtained in the XRD measurements allows us to observe that, with the addition of powdered magnetite in the plastic matrix, an amorphous-crystalline transition is created in the material.

Through the images obtained by SEM, the presence of hematite in the precursor $\left(\mathrm{Fe}_{3} \mathrm{O}_{4}\right)$ and irregular size in the samples is observed. The compounds behave like a soft magnetic material and present changes in the variation of the coercive force, both with the increase in temperature and with the percentage variation of magnetite volume in the polymer matrix. The manufactured material can be used in applications where the compound should be easily detectable.

\section{Keywords}

Epoxy resin, ferrimagnetic, magnetite, composite materials, waster tire. 

magnetita en diferentes proporciones

\section{INTRODUCCIÓN}

Con la continua producción y demanda de automóviles y motocicletas alrededor del mundo y especialmente en los países latinoamericanos, la disposición final de las llantas usadas se ha convertido en un problema ambiental y de salud pública de primer interés [1]. Métodos convencionales, tales como quema y enterrado bajo tierra liberan una gran cantidad de dióxido de carbono y otras sustancias tóxicas que causan cambio climático y graves problemas de contaminación [2]. Por otro lado, las llantas convertidas en basura sin control constituyen focos de infección al promover el anidamiento de fauna nociva, particularmente mosquitos, ratas, arañas y demás especies que buscan lugares oscuros y protegidos para reproducirse. Es bien conocido el hecho de que se tienen dos especies de mosquitos que predominan en los lugares destinados para el acopio de llantas, (Aedes aegypti y Aedes albopictus) que son los principales vectores de la fiebre amarilla y el dengue [3].

Acorde a la compañía MEAM (mediciones del medio ambiente consultoría, Belo Horizonte, MG, Brasil), especialistas en monitoreo ambiental, alrededor de 32 millones de llantas son producidas en Brasil y se estima que de 10 a 15 millones de unidades son desechadas cada año [2]. Países como Colombia y México producen entre 5.3 y 40 millones de llantas que cumplen su vida útil por año, las cuales terminan en andenes, separadores, parques, humedales e incluso junto a las casas [4], [5]. Frente a esta problemática, la atención se ha desplazado a la reutilización de los residuos de llantas con menos contaminación secundaria [6].

En la literatura se pueden encontrar algunos métodos para uso de los residuos de llantas en desuso, a las cuales se les ha extraído el caucho que posteriormente ha sido molido o pulverizado; acerca del uso de las partículas de caucho de neumático, en los estudios realizados podemos encontrar el uso de las partículas de caucho de neumático como un agregado en el concreto, en la fabricación de alfombras, como aislantes de vehículos y campos de juegos [2], [7]. Por otro lado, se pueden encontrar trabajos relacionados con el dopaje de la red polimérica, ya sea con algún tipo de ferrita o mediante la fabricación de compuestos de cauchos magnéticos a través de microondas asistida en la modificación de superficie in situ y procesos de semi-desvulcanización [6].

Polímeros termoestables como la resina epoxi son materiales económicos que curan a temperatura ambiente. Además, son materiales dieléctricos que presentan buena resistencia mecánica, química y a la humedad. Propiedades que varían dependiendo del tipo de resina base, agente de curado, como de los distintos modificadores que pueden añadirse y de las condiciones de polimerización [8]. El uso de este tipo de polímeros ha sido diverso, por ejemplo, la utilización de resina polimérica en la formación de nanopartículas magnéticas de una matriz de sílice, el dopaje de resina epoxi con ferritas de bario y estroncio para la obtención de resinas magnéticas $\mathrm{y}$, por último, se pueden mencionar mecanismos de blindaje electromagnético a través del dopaje de matrices de resina epoxi con uno de los principales elementos de refuerzos en la fabricación de neumáticos como es el negro carbón (CB) [9], [10], [11], [1].

Los métodos más comunes para la fabricación de los compuestos de matriz de plástico y ferritas varían de acuerdo con la naturaleza de los materiales a mezclarse. Los materiales compuestos de matriz de resina epoxi presentan procesos de fabricación más sencillos, donde el material particulado se dispersa en moldes junto con el material termoestable. Por otro lado, la ferrita constituye uno de los mejores materiales magnéticos para ser empleados junto con matrices poliméricas [11]. 

magnetita en diferentes proporciones

Por lo anterior, el objetivo de este trabajo es reportar el proceso de producción de un material compuesto con matriz de resina pre-acelerada, reforzada con magnetita y ripio de llanta, con el fin de buscar un método económico y práctico para la reutilización de las llantas en desuso. La caracterización del compuesto obtenido se llevó a cabo mediante DRX, MEB y estudio de la respuesta magnética.

Con el material fabricado se puede realizar objetos de forma compleja con un excelente comportamiento ante la corrosión ambiental, que pueden ser utilizado en aplicaciones, donde las piezas tienen que moverse o mantenerse en posiciones mediante imanes externos, o en aplicaciones donde el compuesto debe ser fácilmente detectable [12].

\section{PARTE EXPERIMENTAL}

\subsection{Preparación de las muestras}

Para la elaboración del compuesto se emplea resina epoxi como matriz polimérica, la cual previamente fue mezclada con $2 \%$ de catalizador en peso de resina, con el fin de iniciar una reacción química que conlleva a un proceso de curado. El porcentaje de catalizador usado en la matriz polimérica se determinó por medio de experimentos previos, los cuales evidenciaron que este porcentaje en peso era el adecuado para la fabricación de los compuestos. Posteriormente, dicha mezcla entre catalizador y resina es reforzada con dos materiales: caucho pulverizado procedente de llantas en desuso y, como relleno magnético, magnetita pulverizada $\left(\mathrm{Fe}_{3} \mathrm{O}_{4}\right)$. Como regla general, conviene mantener lo más bajo posible el contenido de carga en la matriz polimérica [10], por lo cual se utilizó un porcentaje en volumen de carga que se acoplara a esta condición como se observa en la Tabla 1.

El término carga hace referencia a la mezcla entre caucho pulverizado y magnetita en polvo. Las densidades utilizadas para determinar el porcentaje de volumen son: caucho pulverizado $0.54^{\mathrm{g}} / \mathrm{cm}^{3}$, resina epoxi, $1.11 \mathrm{~g} / \mathrm{cm}^{3}$, magnetita $5.2^{\mathrm{g}} / \mathrm{cm}^{3}$, valores que fueron suministrados por Gestión y Reciclaje Integral de Neumáticos (GRIN) y ficha técnica de resina epoxi. El proceso de curado y pos-curado de las muestras se realizó a temperatura ambiente durante 3 semanas.

\subsection{Caracterización}

La estructura y las fases presentes tanto en los precursores como en el compuesto fueron identificadas por medio de DRX, utilizando un equipo Panalytical X-Pert PRO MPD, del Departamento de Física de la Universidad Nacional de Colombia, sede Bogotá, el cual usa una radiación $\mathrm{Cu}-\mathrm{Ka}$ de $\lambda=1.54$ A. Durante el experimento se definió un intervalo de medición de $10-80^{\circ}$ en la escala de $2 \theta$, con un paso de $0.02^{\circ} \mathrm{y}$ un tiempo de $2 \mathrm{~s}$.

Tabla 1. Designación de las muestras y distribución en porcentajes en volumen. Fuente: [1].

\begin{tabular}{ccccc}
\hline Muestra & \% Vol $\boldsymbol{F e}_{\mathbf{3}} \boldsymbol{O}_{\mathbf{4}}$ & $\begin{array}{c}\text { \% Vol caucho } \\
\text { pulverizado }\end{array}$ & \% vol resina & \% vol de carga en resina \\
\hline Comp 1 & 0 & 34 & 66 & 34 \\
Comp 2 & 3.3 & 33 & 64 & 36.1 \\
Comp 3 & 5.3 & 32.6 & 62.5 & 37.5 \\
Comp 4 & 7.8 & 31.3 & 59.3 & 39.1 \\
Comp 5 & 10.1 & 30.5 & & 40.6 \\
\hline
\end{tabular}



magnetita en diferentes proporciones

El análisis de tamaño y morfología de los precursores y de las muestras se efectuó mediante MEB, utilizando un Microscopio de Barrido Vega 3 TESCAN, del Departamento de Física de la Universidad Nacional de Colombia.

Las propiedades magnéticas fueron estudiadas en curvas de magnetización. La estructura y las fases presentes tanto en los precursores como en el compuesto fueron identificadas por medio de DRX, utilizando un equipo Panalytical X-Pert PRO MPD, del Departamento de Física, de la Universidad Nacional de Colombia, sede Bogotá, el cual usa una radiación $\mathrm{Cu}-\mathrm{Ka}$ de $\lambda=1.54 \AA$. Durante el experimento se definió un intervalo de medición de 10-800 en la escala de $2 \theta$, con un paso de 0.020 y un tiempo de $2 \mathrm{~s}$. El análisis de tamaño y morfología de los precursores y de las muestras se efectuó mediante MEB, utilizando un Microscopio de Barrido Vega 3 TESCAN, del Departamento de Física de la Universidad Nacional de Colombia. Las propiedades magnéticas fueron estudiadas en curvas de magnetización en función del campo aplicado, a través de un equipo VersaLab, Cryogen-free PPMS (Physical Property Measurement System) de Quantum Design, el cual permite la aplicación de campos magnéticos hasta 30000 Oe. en función del campo aplicado, a través de un equipo VersaLab, Cryogenfree PPMS (Physical Property Measurement System) de Quantum Design, el cual permite la aplicación de campos magnéticos hasta 30000 Oe.

\section{RESULTADOS Y DISCUSIÓN}

\subsection{Estructura}

La evaluación del patrón de difracción obtenido en las mediciones de DRX se realizó por medio del programa High Score Plus, usando la base de datos COD_2013, que permite determinar las fases presentes en cada una de las muestras con diferentes proporciones de magnetita en polvo, y de igual forma a los precursores que constituyen los especímenes.

Se estableció que la magnetita en polvo está constituida por dos fases diferentes identificadas como magnetita y hematita, con un porcentaje en peso de $90,2 \%$ y 9,8 $\%$ respectivamente. La magnetita posee una estructura cristalina cúbica centrada en las caras, mientras que la hematita pertenece al sistema cristalino hexagonal [13]. Así mismo, se identificó que la resina epoxi está compuesta en un $100 \%$ de styrene, perteneciente al sistema cristalino ortorrómbico.

El refinamiento Rietveld de los datos experimentales de DRX permitió establecer que el ripio de llanta en polvo está compuesto de dos fases que pertenecen a un sistema cristalino tetragonal. En primer lugar, la fase con fórmula química $\mathrm{Si}_{64.00} \mathrm{O}_{128.00} \mathrm{~N}_{4.16} \mathrm{C}_{45.44} \mathrm{H}_{66.24}$ fue identificada con el nombre de compuesto 4107507, y asociada al código de referencia 96-4107508 , con un porcentaje en peso de fase del $65.9 \%$. En segundo lugar, se obtuvo cristobalite, en un porcentaje en fase de $34.1 \%$, con fórmula química $\mathrm{Si}_{4.00} \mathrm{O}_{8.00}$ asociada al código de referencia 96-9008230, con grupo espacial P 41212 . Cabe señalar que el precursor ripio de llanta en polvo posee otras fases que no son descritas en este trabajo, como las mencionadas en [1], las cuales no fueron posibles identificar mediante esta técnica. Además, las fases no identificadas son muy pequeñas en proporción, por lo cual la contribución magnética de estas fases minoritarias dependerá exclusivamente del porcentaje de elementos magnéticos que posea cada fase. Pero como se observa en [1], las proporciones de estos elementos son muy bajas para afectar las medidas magnéticas.

Los compuestos presentaron cinco fases: cristobalita, styren, hematita, magnetita y por último la fase con fórmula química $\quad \mathrm{Si}_{64.00} \mathrm{O}_{128.00} \mathrm{~N}_{4.16} \mathrm{C}_{45.44} \mathrm{H}_{66.24} . \quad \mathrm{El}$ método utilizado para determinar la estequiometria química de los compuestos como de los precursores fue a través de la 
comparación de las pdfs ubicadas en la base de datos COD_2013 por medio del software X'Pert Highscore plus elaborado por Panalytical B. V. Los porcentajes de las fases presentes en los especímenes fue determinado por medio de refinamiento Rietveld (ver tabla 2).

\subsection{Comparación de los patrones de difracción}

En la Fig. 1 se presenta el análisis de los difractogramas experimentales, tanto de los precursores como de las muestras, el cual fue efectuado con el fin de observar el comportamiento de dichos precursores después de ser conformados en un compuesto.

Los patrones de difracción de cada una de las muestras se pueden ver en la Fig.1. De las Figuras 1a y 1 b se ve que la resina y el caucho pulverizado presentan perfiles similares.

En muchos casos, no tenemos una estructura de cristal disponible, el estado de la materia puede inferirse de la apariencia del patrón. En la Fig. 2c, los patrones de polímero consisten en múltiples picos agudos consistentes con la «difracción» de una red cristalina. En la Fig. 2a, los patrones tienen características muy amplias consistentes con la «dispersión incoherente» de un sólido amorfo. Los patrones de la Fig. 2b son más complejos, exhibiendo una mezcla por lo cual el material se comporta como un material semi-cristalino [14]. Con base a lo anterior, se puede decir que al mezclar la resina epoxi con el ripio de llanta en polvo, la difusión es muy completa, es decir, todo lo que era cristalino en el ripio de llanta se difundió y se transformó en amorfo (ver Fig. 1a y 1b).

Por otro lado, los difractogramas de las muestras cuyo contenido de ferrita varía de un $3.3 \%$ a un $10.1 \%$ en volumen de magnetita, revelan que al combinar resina epoxi, magnetita en polvo y ripio de llanta se conforma un compósito semicristalino, donde hay una matriz amorfa y unas partículas cristalizadas, identificadas como magnetita y hematita. De la misma manera, se observa una progresiva disminución de la zona amorfa que hace parte del relieve comprendido en la región $2 \theta$ de 15.770 y 23.090 . A partir del $5.33 \%$ de relleno de magnetita en la región amorfa se evidencia un incremento paulatino de picos bien formados pertenecientes a la fase cristalina (magnetita y hematita) ubicados en la región $2 \theta$ de: 30.090, 35.440, 53.420, 56.940 y 62.520 .

Se puede inferir, entonces, que hay una transición amorfo-cristalino entre las muestras al ir aumentando el contenido en volumen de magnetita. Esto ocurre debido al agregado de la fase cristalina $\mathrm{Fe}_{3} \mathrm{O}_{4}$ que emigra en la fase amorfa del material, reduciendo así los dominios amorfos de la muestra Comp1 (ripio de llanta en polvoresina epoxi). El difractograma también indica que la estructura de $\mathrm{Fe}_{3} \mathrm{O}_{4}$ se mantiene[15].

Tabla 2. Fases pertenecientes a los compuestos en función del porcentaje de $\mathbf{F e}_{3} \mathbf{O}_{\mathbf{4}}$ en la matriz polimérica.

Fuente: [1].

\begin{tabular}{|c|c|c|c|c|c|c|}
\hline & & \multicolumn{5}{|c|}{ Fase } \\
\hline Muestra & \%vol de $\mathrm{Fe}_{3} \mathrm{O}_{4}$ & Magnetita & Hematita & Styrene & Cristobalite & $\mathrm{Si}_{64.00} \mathrm{O}_{128.00} \mathrm{~N}_{4.16} \mathrm{C}_{45.44} \mathrm{H}_{66.24}$ \\
\hline Comp 2 & 3.3 & 18.7 & 0.8 & 43 & 21.7 & 15.7 \\
\hline Comp3 & 5.3 & 10.7 & 0,8 & 56.6 & 21 & 10.8 \\
\hline Comp 4 & 7.8 & 26 & 2 & 42 & 0.2 & 29.9 \\
\hline Comp 5 & 10.1 & 24.6 & 1.8 & 38 & 0.4 & 35.1 \\
\hline
\end{tabular}


Caracterización magnética de material compuesto con matriz de resina epoxi y llanta en desuso reforzado con magnetita en diferentes proporciones

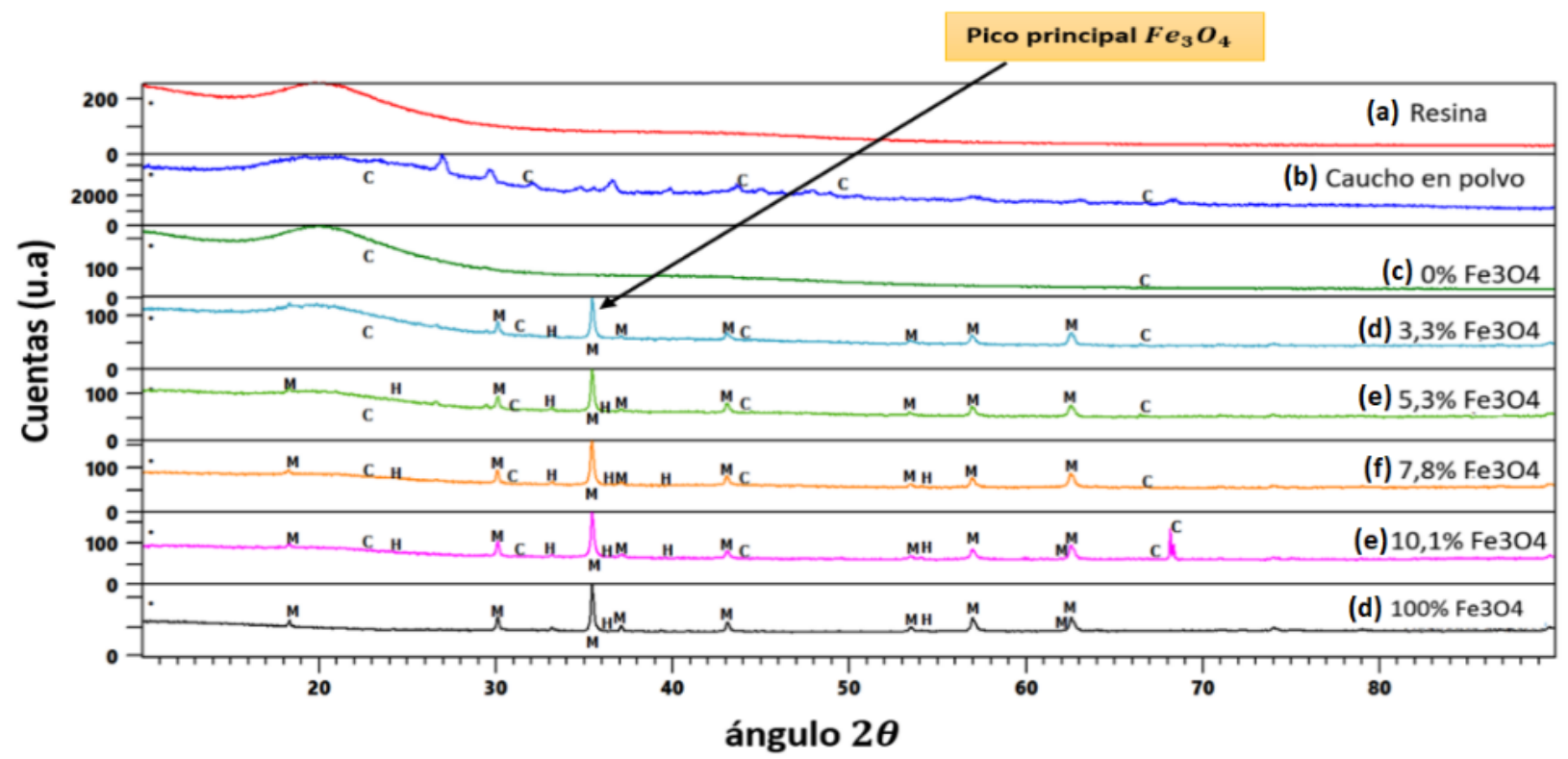

Fig.1. Patrones de difracción de los precursores: a) $100 \%$ de resina b) $100 \%$ de caucho en polvo y h) $100 \%$ magnetita en polvo. Patrones de difracción para todas las muestras con diferentes porcentajes en volumen de magnetita en polvo; c) Comp 1 con un $0 \%$ de magnetita, d) Comp 2 con un $3.3 \%$ de ferrita, e) Comp 3 con un 5.3 $\%$ de ferrita, f) Comp 4 con un $7.8 \%$ de ferrita, g) Comp 5 con un10.1\% de ferrita h) $100 \% \mathrm{Fe}_{3} \mathrm{O}_{4}$. Fuente:[1].
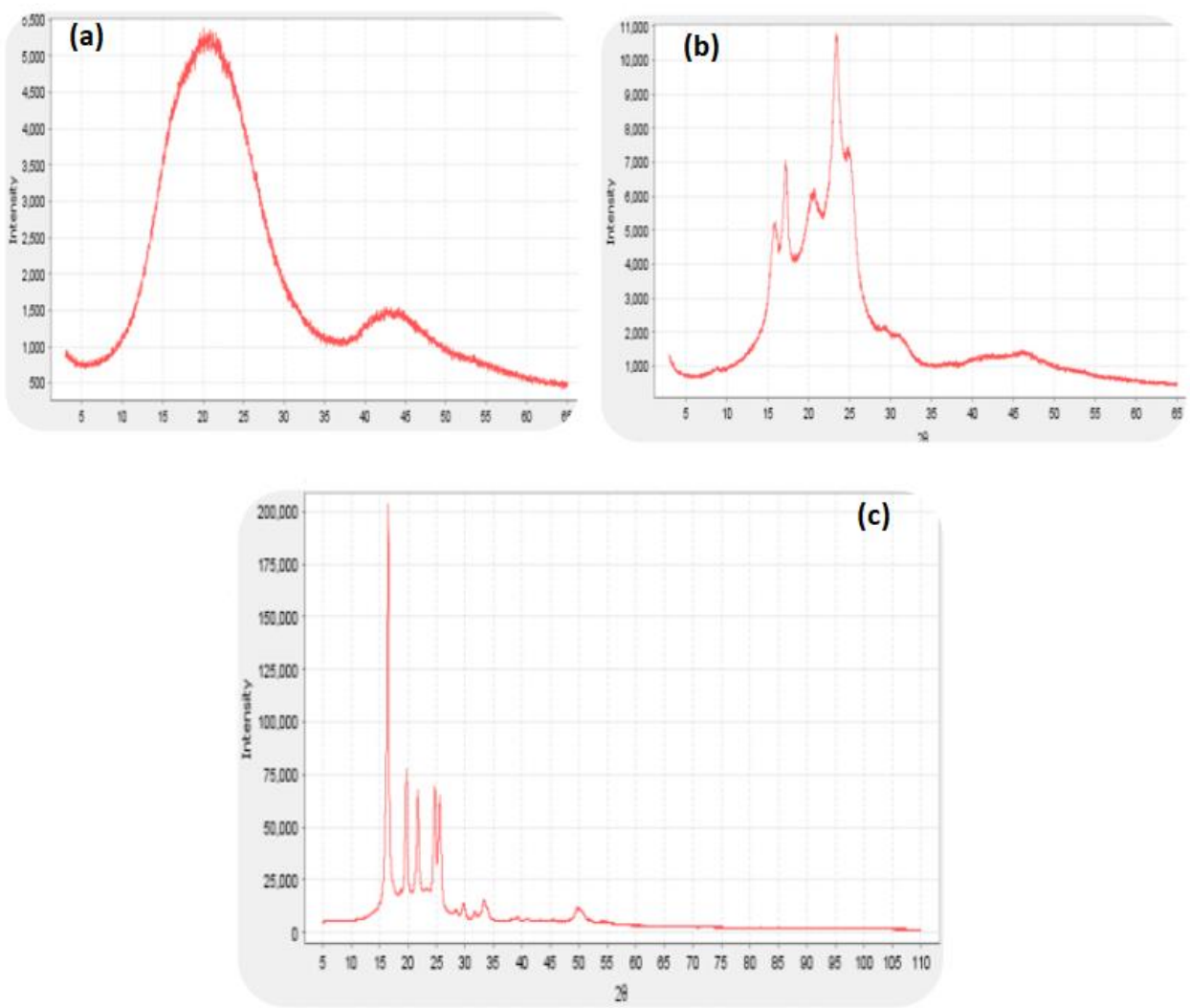

Fig. 2. patrones representativos de DRX: a) patrón de difracción de un polímero amorfo b) patrón para un polímero semi-cristalino c) patrón de difracción para un polímero cristalino. Fuente: [15] 
A partir de los difractogramas queda en evidencia que la resina, ripio de llanta en polvo y la magnetita nunca reaccionan entre sí conformando una nueva fase y por ende no se observan nuevos picos de difracción [13].

\subsection{Caracterización morfológica}

Con el fin de evaluar la morfología del compuesto, se tomaron imágenes de MEB tanto a las muestras con diferentes porcentajes en volumen de magnetita como a los precursores. En la Fig. 3 se observan micrografías de la magnetita en polvo, la cual fue usada como material de refuerzo en la matriz polimérica. De las imágenes se distinguen diferentes rangos de tamaño de partícula de irregular forma y con una baja relación de aspecto, los resultados anteriores son similares a los reportados en [14].

A partir del análisis se estableció que los granos presentes en la figura 3 procedentes de la magnetita en polvo poseen tamaños entre $0.9 \pm 0.3$ y $4.6 \pm 0.2$ $\mu \mathrm{m}$. Por otro lado, las imágenes muestran las diferencias entre la hematita y la magnetita, ambas fases presentes en el precursor en polvo. La hematita se

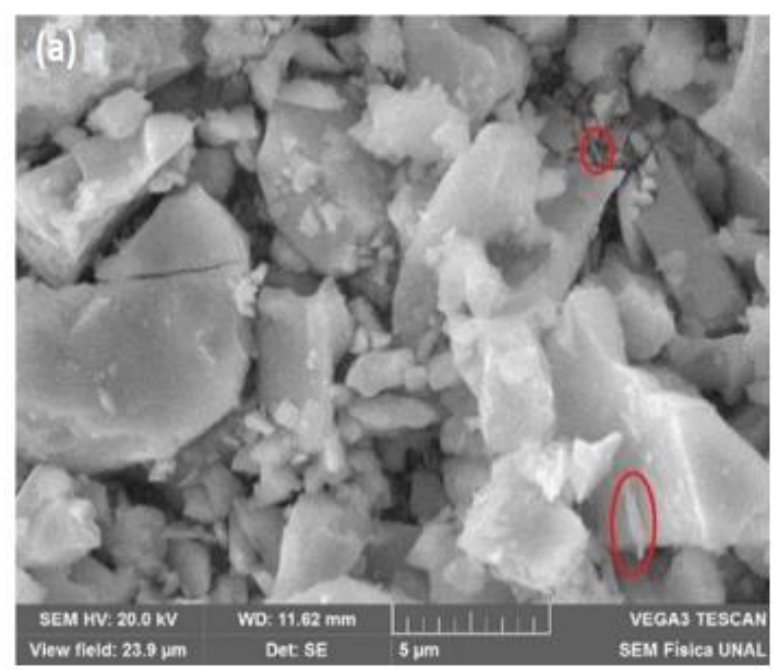

caracteriza por su morfología laminar tipo aguja y su color rojo brillante, aunque la morfología de los granos se puede ver influenciada por el método de obtención del material, mientras que la magnetita presenta una morfología más volumétrica que se aproxima a un tipo de grano esférico de forma irregular de color negro [13].

En las imágenes MEB de la Fig. 3, pertenecientes al precursor magnetita en polvo, se puede observar algunos granos en forma de aguja encerrados en un círculo rojo, esta forma característica de grano hace referencia a presencia de hematita en el precursor magnetita en polvo, lo cual concuerda con los resultados encontrados por difracción de rayos $\mathrm{x}$ para este precursor.

La Fig. 4 ejemplifica la morfología superficial del ripio de llanta en polvo. En las imágenes se observa que la morfología del caucho pulverizado es irregular con rugosidad superficial y con zarcillos o riso que se proyectan desde la superficie formando cavidades en la superficie del material [16]. Por el método de los interceptos se determinó que el tamaño promedio del grano se encuentra entre 1.4 $\pm 0.3 \mathrm{y} 3.1 \pm 0.2 \mu \mathrm{m}$.

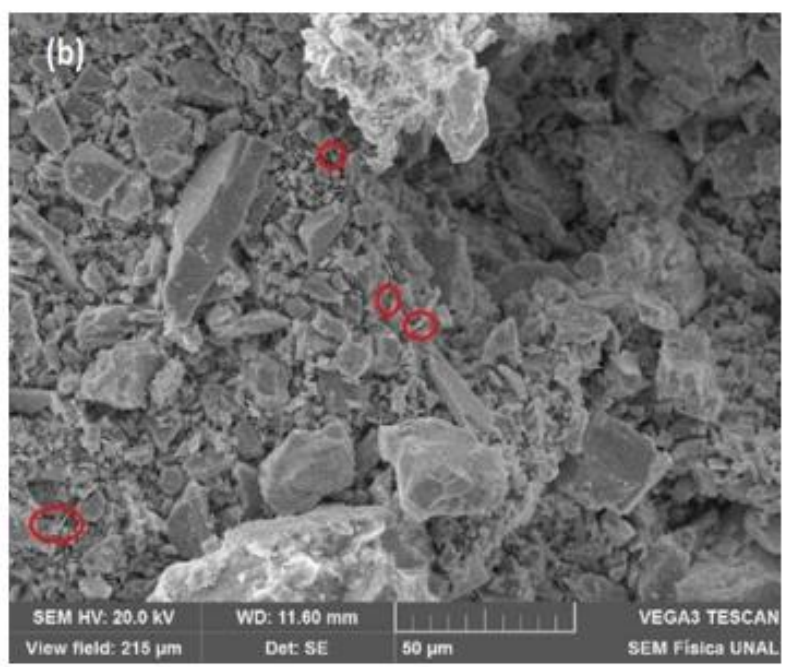

Fig. 3. Imágenes MEB del precursor magnetita $\left(\mathrm{Fe}_{3} \mathrm{O}_{4}\right)$ tomadas con diferentes escalas de longitud a) $\mathbf{5} \boldsymbol{\mu m}$ b) $\mathbf{5 0}$ $\boldsymbol{\mu m}$, en círculos de color rojo los cristales laminares de hematita. Fuente: [1]. 

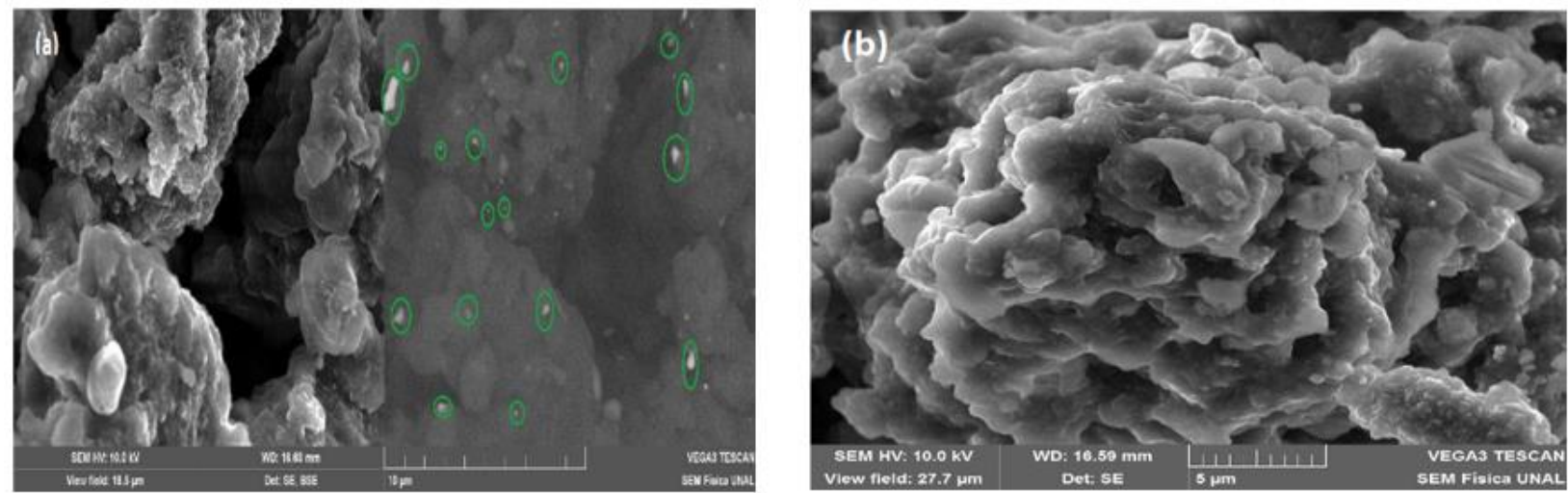

Fig. 4. Imágenes MEB del precursor ripio de llanta en polvo tomadas a dos escalas diferentes de longitud a) $5 \mu m$ y b) $10 \mu \mathrm{m}$. En recuadro derecho de la imagen a), se observa cristales de hierro encerrados en círculos de color verde. Fuente: [1].

Cabe recalcar que se presentan partículas brillantes dentro del material, como se observa en la parte derecha de la imagen de la Figura 3a, las cuales, de acuerdo con la caracterización por fluorescencia, se sugiere que están constituidas por hierro. Dichas partículas son consideradas impurezas, debido a que en la literatura no se encuentra reportado que el caucho pulverizado procedente de llantas en desuso posea estas partículas, por lo cual un posible origen de estas partículas se encuentra en el proceso de obtención del caucho en polvo, debido a que el método es mecánico. De manera que en el desarrollo de molienda pudo haber contacto con el enmallado que poseen las llantas y de esta manera quedaron elementos de hierro en el caucho obtenido en forma de polvo.

La Fig. 5 presenta las imágenes MEB pertenecientes a las muestras. En las mismas no se puede distinguir claramente la fase perteneciente al ripio de llanta y la resina epoxi, ya que estas se interceptan entre sí. Además, se observa que las muestras presentan una microestructura no homogénea de forma irregular. Por otro lado, se evidencia que las incrustaciones de partículas de magnetita tienen una gran superficie, lo cual aumenta el contacto con la matriz caucho epoxi (ver Fig. 5c). Esto a su vez puede aumentar la resistencia del caucho epoxi debido al enclavamiento mecánico que surge del aumento del contacto entre las partículas del refuerzo y la matriz epoxi-caucho, por lo cual la interface entre la matriz y el refuerzo están bien creadas [17].

Se estimó que el tamaño del grano para las muestras se encuentra entre $0.3 \pm 0.1 \mathrm{y}$ $2.5 \pm 0.5 \mu \mathrm{m}$, lo cual indica que los tamaños del grano de las muestras no son uniformes. Además, algunas de las partículas se vieron más grandes que su tamaño real, que es una consecuencia de la aglomeración en ciertas áreas [17].

$\mathrm{Al}$ igual que en [18], se observa que los granos de magnetita en polvo penetraron profundamente en la matriz polimérica sin dejar vacío (ver Fig. 5c y 5d). La ausencia de huecos implica que no se produjo oxidación dentro del ripio de llanta en polvo durante la formación de las muestras. Se debe agregar que las distribuciones de los granos de magnetita en polvo son uniformes en los compuestos (ver Fig. 5e), lo cual asegura que el flujo magnético fluya uniformemente a través del compuesto [18].

\subsection{Caracterización magnética}

A las muestras Comp 1, Comp2, Comp 3, Comp 4 y Comp 5 se les realizó curvas de histéresis magnética a $50 \mathrm{~K}, 120 \mathrm{~K}$ y 
300K, bajo la aplicación de un campo magnético externo comprendido entre 30000 Oe y 30000 Oe.

Los ciclos de histéresis en función del porcentaje de volumen de magnetita para diferentes temperaturas para las muestras obtenidas se presentan en las Fig. 5. Los parámetros magnéticos característicos Magnetización de saturación (Ms), Magnetización de remanente (Mr) y Campo coercitivo (Hc) de los compuestos se muestran en la Tabla 3.

Las curvas de histéresis para las temperaturas 50,120 y $300 \mathrm{~K}$ que presentan las muestras Comp 1 hasta Comp 4 son curvas características de un material ferrimagnético (ver Fig. 6). En el caso de la muestra designada como Comp 1 (0 \% vol. magnetita) para temperaturas comprendidas entre 120 y $300 \mathrm{~K}$, el espécimen se comporta como un material ferromagnético, debido a los elementos presentes en el precursor ripio de llanta como lo son el Zinc y el hierro, elementos determinados por fluorescencia (ver Fig. 6).

Cabe señalar que la muestra Comp1 presenta valores de magnetización remanente y magnetización de saturación pequeños, tanto para $120 \mathrm{~K}$ como para $300 \mathrm{~K}$, debido a las propiedades magnéticas de las impurezas presentes en el material, las cuales han sido nombradas con anterioridad en este documento.

A partir de la Tabla 3 se puede inferir que con el incremento en \%vol de $\mathrm{Fe}_{3} \mathrm{O}_{4}$ tanto los valores de magnetización remanente como la magnetización de saturación se hacen mayores. Estos resultados coinciden con los obtenidos en [13], y se puede explicar a través de la interacción probable entre las propiedades magnéticas de las partículas de magnetita dentro de la matriz polimérica cauchoepoxi, como de las impurezas presentes en el ripio de llanta pulverizado. Además, se determina que la magnetización de saturación del compuesto es linealmente dependiente de la fracción de masa de relleno según la relación: $\mathrm{MS}=\mathrm{MF} \times \mathrm{WF}$, donde MF y WF son la magnetización de carga y la fracción de peso de carga en el compuesto [19].

Es importante decir que el área bajo la curva del ciclo de histéresis para materiales ferromagnéticos corresponde a la energía disipada o perdidas por histéresis; en el caso de los materiales magnéticos blandos esta energía de perdida es baja, por tanto, se produce menos calor para disipar [21].

Tabla 3. Parámetros magnéticos de las muestras a diferentes temperaturas: Magnetización de saturación (Ms), Magnetización de remanente (Mr), Campo coercitivo (Hc). Fuente: [1].

\begin{tabular}{|c|c|c|c|c|c|c|c|c|c|}
\hline \multicolumn{4}{|c|}{ Propiedades de la muestra a $300 \mathrm{~K}$} & \multicolumn{3}{|c|}{$\begin{array}{l}\text { Propiedades de la muestra a } \\
\qquad 120 \mathrm{~K}\end{array}$} & \multicolumn{3}{|c|}{$\begin{array}{l}\text { Propiedades de la muestra a } \\
50 \mathrm{~K}\end{array}$} \\
\hline Muestr & $\mathrm{M}_{\mathrm{s}}$ & $\mathrm{M}_{\mathrm{r}}$ & $\mathrm{H}_{\mathrm{c}}$ & $\mathrm{M}_{\mathrm{s}}$ & $\mathrm{M}_{\mathrm{r}}$ & $\mathrm{H}_{\mathrm{c}}$ & $\mathrm{M}_{\mathrm{s}}$ & $\mathrm{M}_{\mathrm{r}}$ & $\mathrm{H}_{\mathrm{c}}$ \\
\hline & $(\mathrm{emu} / \mathrm{g})$ & $(\mathrm{emu} / \mathrm{g})$ & $\pm 5 \times 10^{-4} T$ & $(\mathrm{emu} / \mathrm{g})$ & $(\mathrm{emu} / \mathrm{g})$ & $\pm 5 \times 10^{-4}$ & (emu/g) & $(\mathrm{emu} / \mathrm{g})$ & $\pm 5 \times 10^{-}$ \\
\hline Comp 1 & $1.32 \pm 0.03$ & $\begin{array}{c}0.02 \\
\pm 0.04\end{array}$ & 0.0022 & $\begin{array}{c}1.36 \\
\pm 0.03\end{array}$ & $\begin{array}{c}2.91 \\
\pm 0.06\end{array}$ & 0.0032 & & & \\
\hline Comp 2 & $\begin{array}{l}15.24 \\
\pm 0.03\end{array}$ & $\begin{array}{c}1.14 \\
\pm 0.02\end{array}$ & 0.0064 & $\begin{array}{c}16.0 \\
\pm 0.03\end{array}$ & $\begin{array}{c}1.20 \\
\pm 0.02\end{array}$ & 0.0070 & $\begin{array}{c}16.4 \\
\pm 0.03\end{array}$ & $\begin{array}{c}1.63 \\
\pm 0.03\end{array}$ & 0.0167 \\
\hline Comp 3 & $\begin{array}{c}22.91 \pm \\
0.05\end{array}$ & $\begin{array}{c}1.33 \\
\pm 0.03\end{array}$ & 0.0081 & $\begin{array}{c}24.2 \\
\pm 0.05\end{array}$ & $\begin{array}{c}1.42 \\
\pm 0.03\end{array}$ & 0.0082 & $\begin{array}{c}24.7 \\
\pm 0.05\end{array}$ & $\begin{array}{c}2.46 \\
\pm 0.05\end{array}$ & 0.0164 \\
\hline Comp 4 & $\begin{array}{c}31.11 \pm \\
0.06\end{array}$ & $\begin{array}{c}1.89 \\
\pm 0.04\end{array}$ & 0.0080 & $\begin{array}{c}32.8 \\
\pm 0.07\end{array}$ & $\begin{array}{c}2.07 \\
\pm 0.04\end{array}$ & 0.0087 & $\begin{array}{c}33.0 \\
\pm 0.07\end{array}$ & $\begin{array}{c}3.52 \\
\pm 0.07\end{array}$ & 0.0169 \\
\hline Comp 5 & $\begin{array}{c}35.10 \pm \\
0.07\end{array}$ & $\begin{array}{c}2.06 \\
\pm 0.04\end{array}$ & 0.0078 & $\begin{array}{c}37.1 \\
\pm 0.07\end{array}$ & $\begin{array}{c}2.21 \\
\pm 0.04\end{array}$ & 0.0089 & $\begin{array}{c}37.3 \\
\pm 0.07\end{array}$ & $\begin{array}{c}4.00 \\
\pm 0,01\end{array}$ & 0.0170 \\
\hline
\end{tabular}


Caracterización magnética de material compuesto con matriz de resina epoxi y llanta en desuso reforzado con magnetita en diferentes proporciones
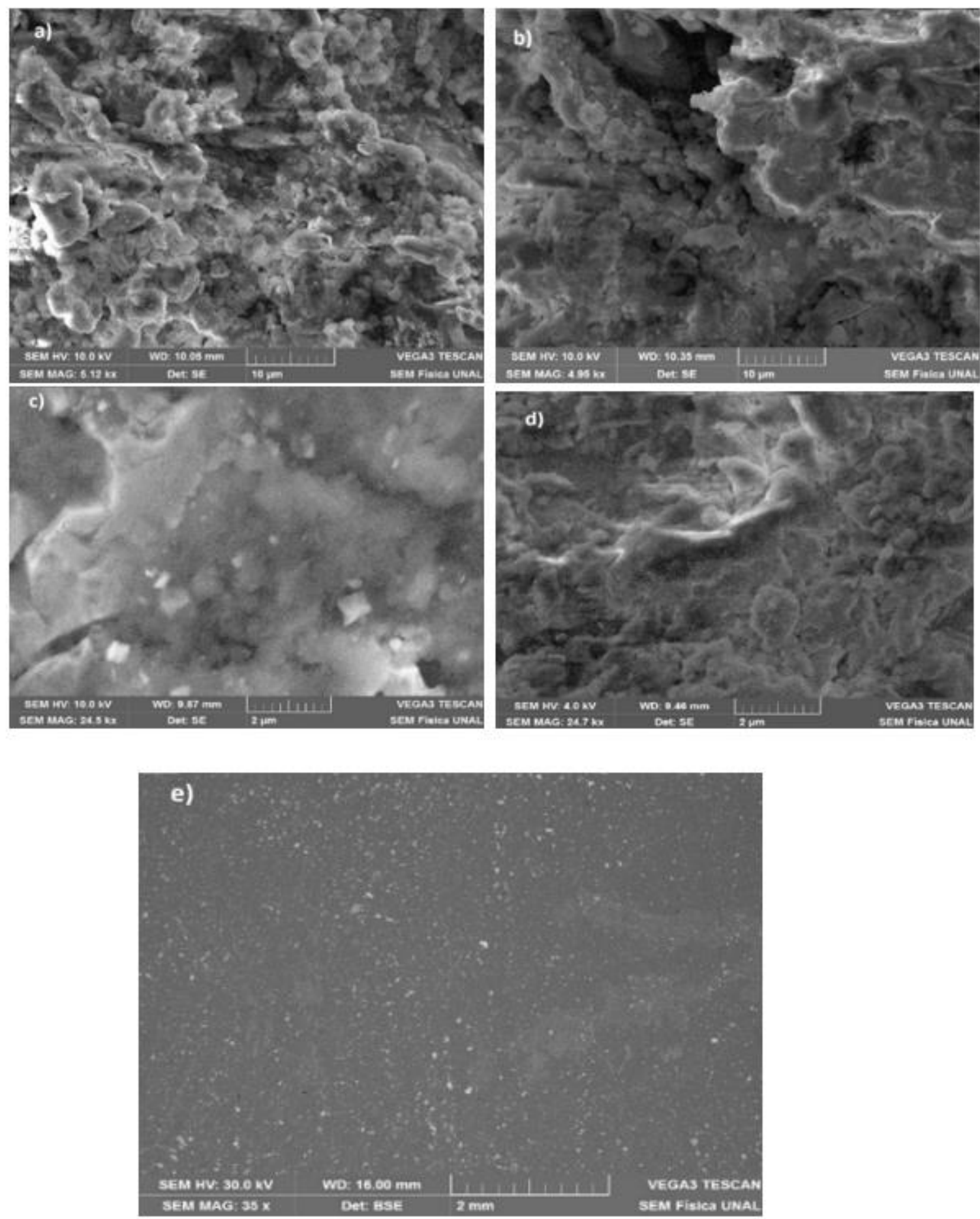

Fig. 5. Imagen MEB de las muestras estudiadas con diferentes $\%$ vol de $\mathrm{Fe}_{3} \mathrm{O}_{4}$ (Comp 1-Comp 5), tomadas a una escala de longitud de $2 \mu \mathrm{m}$ : A) $3.3 \%$ a $5.1 \mathrm{kx}$, B) $5.3 \%$ a $49 \mathrm{kx}$, C) $7.8 \%$ a $24 \mathrm{kx}$, D) $10.1 \%$ a $24 \mathrm{kx}$ E) 3.3 a $35 \mathrm{x}$. Fuente: [1]. 

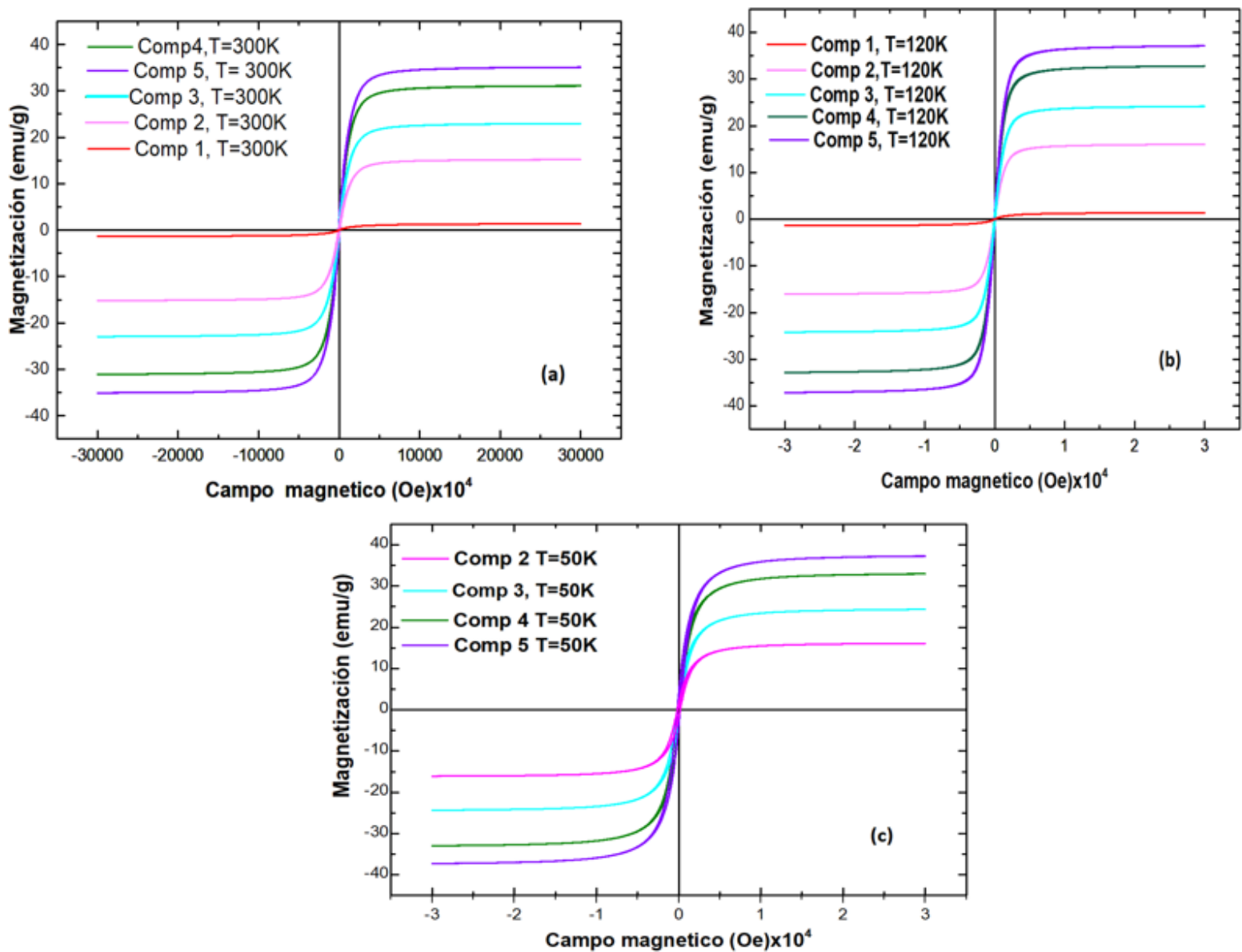

Fig. 6. Curvas de histéresis tomadas a diferentes temperaturas en función de un campo magnético para cada una de las muestras: a) $300 \mathrm{~K}$, b) $120 \mathrm{~K}$, c) $50 \mathrm{~K}$. Fuente: [1].

Un material magnético blando es aquel que es fácil de imanar y desimanar, a diferencia de un material magnético duro. Para que un material ferromagnético sea blando, su ciclo de histéresis debe tener una fuerza coercitiva tan pequeña como sea posible, lo cual permite que el material se imane fácilmente y tenga una alta permeabilidad magnética [22].

Los valores de coercitividad para los compuestos a temperatura ambiente disminuyen con el incremento de ferrita en el compuesto. Estos valores son similares a los reportados en [18]. Por consiguiente, el aumento en Hc en las muestras con menor contenido de relleno indica que la matriz polimérica es resistente a la alineación del momento magnético de la carga. Por lo tanto, los compuestos con menor contenido de relleno apenas se desmagnetizan en comparación con aquellos compuestos con mayor carga de relleno [15]; caso contrario ocurre a temperaturas bajas donde la coercitividad aumenta con el incremento de $\mathrm{Fe}_{3} \mathrm{O}_{4}$ en la matriz plástica.

Se debe agregar que la fuerza coercitiva en las muestras disminuye con el incremento de la temperatura en razón del tamaño de grano que disminuye, ya que la coercitividad se incrementa cuando el tamaño de grano crece, debido que los defectos introducidos por la frontera de grano disminuyen por unidad de volumen [20]. 

magnetita en diferentes proporciones

Además, se debe anotar que las curvas de histéresis para las muestras a diferentes temperaturas manifiestan que los compuestos son materiales magnéticos blandos, debido a que poseen ciclos de histéresis estrechos con pequeñas fuerzas coercitivas (ver Fig. 6).

La gama de aplicaciones de los materiales magnéticos crece con la mejora de sus propiedades magnéticas, mecánicas, eléctricas y térmicas. En comparación con los materiales magnéticos blandos clásicos, los materiales magnéticos blandos modernos tienen una tecnología óptima de fabricación al obtener las propiedades asumidas que permiten miniaturizar, simplificar y reducir los costos de los dispositivos. Por eso, es de gran importancia optimizar el proceso tecnológico de obtención de materiales [23]. De ahí que métodos como el presentado en este trabajo se hacen significativos, las aplicaciones sugeridas para los compuestos magnéticos obtenidos en esta investigación son multiplicadores de flujo, los transductores de otras propiedades (núcleos de sensores) [24], y entre otros como los mencionados en la referencia [23].

$\mathrm{Al}$ realizar gráficas entre temperatura y magnetización remanente y de magnetización de saturación, se observa de estas gráficas, que la magnetización remanente $(\mathrm{M} r)$ y la magnetización de saturación (Ms) disminuyen con la elevación de la temperatura, como indican las Fig. $7 \mathrm{a}$ y $7 \mathrm{~b}$.

Lo anterior se debe al hecho que al incrementar la temperatura el número de partículas en estado superparamagnético se aumentan y en consecuencia la medida de los valores de $M s$ y $\mathrm{Mr}$ disminuyen. En muestras que poseen partículas multidominio, los valores de $M s$ y $\mathrm{Mr}$ tienden también a disminuir, dado que las configuraciones geométricas de los momentos magnéticos tienden a disminuir la energía total del sistema [25]. Cabe resaltar que las propiedades magnéticas evidenciadas en este trabajo son similares a los reportados en [12]-[30], donde se trabaja con materiales idénticos a los utilizados como precursores en este trabajo.

\section{CONCLUSIONES}

El método utilizado permitió la fabricación del compuesto a base de ripio de llanta en desuso y magnetita en polvo, usando resina epoxi como matriz polimérica. El análisis por DRX permite concluir que el ripio de llanta posee impurezas de hierro debido al método de obtención del material. Con el incremento de la magnetita en polvo en la matriz plástica, la zona amorfa perteneciente al polímero caucho-epoxi disminuye, de esta manera se pasa de un material amorfo a un material semicristalino. Frente a las pruebas magnéticas realizadas a los compuestos, se puede deducir que las curvas de histéresis en función del campo aplicados a diferentes temperaturas exhiben que las muestras Comp 2 a Comp 4 son curvas características de un material ferrimagnético. Todas las muestras a diferentes temperaturas se comportan como materiales magnéticos blandos, debido a que poseen ciclos de histéresis estrechos con pequeñas fuerzas coercitivas. Con el incremento de magnetita en la matriz se presenta un incremento de la saturación. Con el aumento de la temperatura las muestras presentan una disminución en magnetización de saturación. Con el aumento de la temperatura las muestras presentan una disminución en magnetización de saturación. La fuerza coercitiva presenta una respuesta independiente de la carga de ferrita presente en la matriz caucho-epoxi. 
Caracterización magnética de material compuesto con matriz de resina epoxi y llanta en desuso reforzado con magnetita en diferentes proporciones
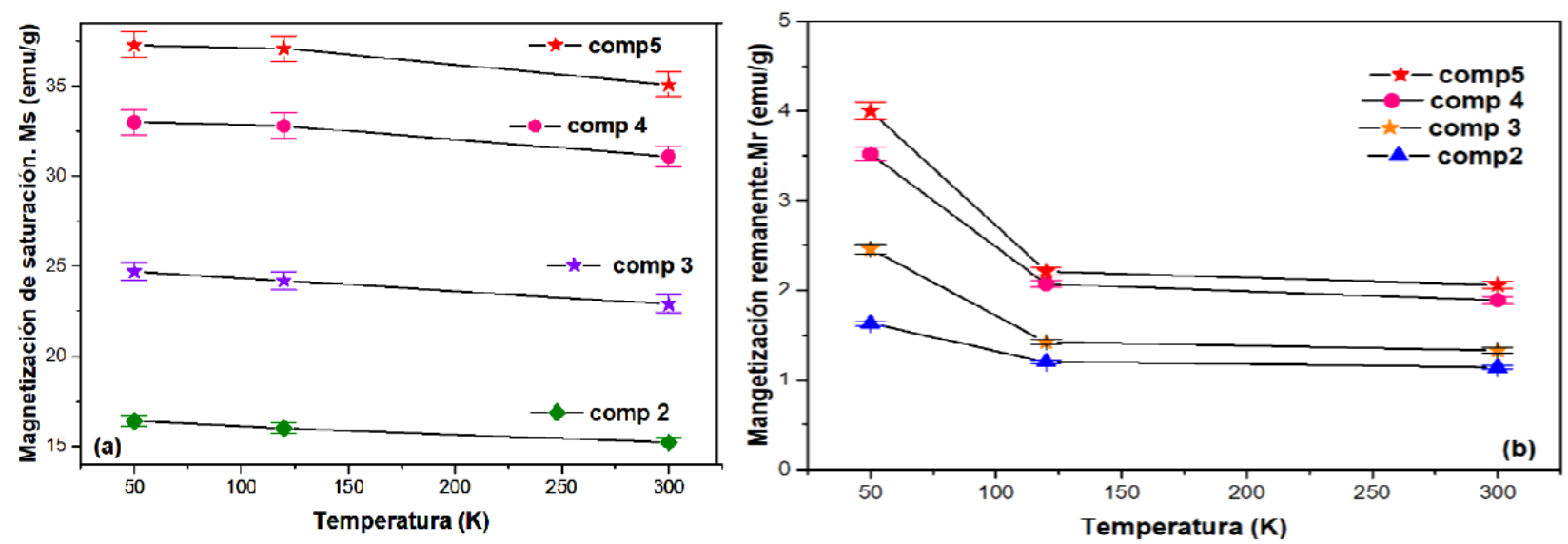

Fig. 7. a) Magnetización de saturación para las muestras a diferentes \% vol de $\mathrm{Fe}_{3} \mathrm{O}_{4}$ vs temperatura b) Magnetización remanente para las muestras a diferentes \% vol de $\mathrm{Fe}_{3} \mathrm{O}_{4}$ vs temperatura. Fuente: [1].

El material fabricado se puede realizar objetos de forma compleja con un excelente comportamiento ante la corrosión ambiental, que pueden ser utilizado en aplicaciones, donde las piezas tienen que moverse o mantenerse en posiciones mediante imanes externos, o en aplicaciones donde el compuesto debe ser fácilmente detectable.

\section{AGRADECIMIENTOS}

El trabajo realizado fue gracias a colaboración del grupo físico de nuevos materiales (GFNM) suscrito a la Universidad Nacional de Colombia.

\section{REFERENCIAS}

[1] F. E. S. Tacumá, "Producción y caracterización de materiales compuestos con matrices de resina epoxi reforzados con ripio de llanta y magnetita en diferentes proporciones," Universidad Nacional de Colombia, 2018.

[2] N. Segre and I. Joekes, "Use of tire rubber particles as addition to cement paste," Cem. Concr. Res., vol. 30, no. 9, pp. 1421-1425, Sep. 2000.

[3] D. A. V. Guerrero and J. C. Barbosa, "Diseño de un plan operativo de recolección, almacenamiento y de gestión para el aprovechamiento de las llantas en desuso en el casco urbano del municipio de ocaña,"
Universidad Francisco de Paula Santander Ocaña, 2012.

[4] L. Gómez, "Comienza era de vías que se harán con llantas usadas”, El Tiempo, 2016. [Online]. Available: http://www.eltiempo.com/archivo/documento/ CMS-16483926. [Accessed: 11-Nov-2018]

[5] J. Campoamor, "Cementerios de neumáticos: un problema global," DW Made for minds, 2016. [Online]. Available: https://www.dw.com/es/cementerios-deneumáticos-un-problema-global/a-19278585. [Accessed: 11-Nov-2018].

[6] J. Liu, P. Liu, X. Zhang, P. Lu, X. Zhang, and M. Zhang, "Fabrication of magnetic rubber composites by recycling waste rubber powders via a microwave-assisted in situ surface modification and semidevulcanization process," Chem. Eng. J., vol. 295, no. 295, pp. 73-79, Jul. 2016.

[7] J. Esteve, "Reciclaje de Neumáticos: procesos y usos," 2012. [Online]. Available: https://reciclajeverde.wordpress.com/2012/06/ 26/reciclaje-de-neumaticos-procesos-y-usos/. [Accessed: 11-Nov-2018].

[8] E. A. González, "Expoxidica." [Online]. Available:

http://contenidos.educarex.es/mci/2009/43/TE MA2/resina_epoxidicas.pdf.

[9] S. M. Montemayor, L. A. G. Cerda, and J. R. T. Lubián, "Uso de una resina polimérica en la formación de nanopartículas magnéticas dentro de una matriz de sílice," Superf. y vacio, vol. 17, no. 2, pp. 21-24, 2004.

[10] C. U. L. Oliveros and D. D. M. Ordóñez, "Obtención, evaluación y aplicación de resinas de poléster magnéticas," Univ. Indus. de Santander, Bucaramanga, Colombia, 2004

[11] A. O. Garzón-Posada, D. A. Landínez-Téllez, J. Roa-Rojas, and J. Ramos-Barrado, 
Caracterización magnética de material compuesto con matriz de resina epoxi y llanta en desuso reforzado con magnetita en diferentes proporciones

"Materiales compuestos de matriz polimérica usados para el blindaje de interferencia electromagnética," Cienc. e Ing. Neogranadina, vol. 27, no. 1, pp. 5-26, Jan. 2017.

[12] R. Mangnus, "Processing and Properties of magnetite -rubbers blends," J-Global, vol. 56, no. 6, pp. 322-329, 2003.

[13] A. O. G. Posada, "Síntesis y caracterización de un material compuesto a base de polietileno de alta densidad y magnetita pulverizada," Universidad Nacional de Colombia, 2015.

[14] ICDD, "How to Analyze Polymers Using Xray Diffraction." [Online]. Available: https://webcache.googleusercontent.com/sear ch?q=cache:PNKaKuOVnAMJ:https://www.r esearchgate.net/profile/Imad_Disher2/post/C an_anyone_help_me_determine_the_percent age_crystallinity_of_an_amorphous_polymer _based_on_XRD_data2/attachment/5b2a7a99 b53d2f892896e.

[15] I. Kong, S. Hj Ahmad, M. Hj Abdullah, D. Hui, A. Nazlim Yusoff, and D. Puryanti, "Magnetic and microwave absorbing properties of magnetite-thermoplastic natural rubber nanocomposites," J. Magn. Magn. Mater., vol. 322, no. 21, pp. 34013409, Nov. 2010.

[16] M. Myhre and D. A. MacKillop, "Rubber Recycling," Rubber Chem. Technol., vol. 75, no. 3, pp. 429-474, Jul. 2002.

[17] A. B. Irez, E. Bayraktar, and I. Miskioglu, "Design and Mechanical-Physical Properties of Epoxy-Rubber Based Composites Reinforced with Nanoparticles," Procedia Eng., vol. 184, pp. 486-496, 2017.

[18] Ubaidillah et al., "A new class of magnetorheological elastomers based on waste tire rubber and the characterization of their properties," Smart Mater. Struct., vol. 25, no. 11, p. 115002 , Nov. 2016.

[19] A. O. Garzón, D. A. Landínez, J. Roa-Rojas, F. E. Fajardo-Tolosa, G. Peña-Rodríguez, and C. A. Parra-Vargas, "Production and structural, electrical and magnetic characterization of a composite material based on powdered magnetite and high density polyethylene," Rev. la Acad. Colomb. Ciencias Exactas, Físicas y Nat., vol. 41, no. 159, pp. 154-167, 2017.

[20] J. Prado, C. Calle, F. Cuéllar, and J. M. Caicedo, "Efecto de La Temperatura de deposición Sobre la Estructura Y Respuesta Magnética de Películas Delgadas de Ferritas de Nizn," Rev. Colomb. Física, vol. 39, no. 2, pp. 419-422, 2007.

[21] D. Horacio, E. Guillermo, A. Rossi, R. Bocero, M. Frechero, and P. di Pratula, "Uso de Materiales Magnéticos Blandos Nanocristalizados en Estator de Generador modular con Imanes Permanentes de flujo Transversal para Turbina Eólica," in Energías Sustentables en Bahía Blanca, 2014, p. 11.

[22] Kimerius, "Materiales Magnéticos." [Online]. Available:

kimerius.com/app/download/5783167773/Mat eriales+magnéticos.pdf\%0A.

[23] B. Ziębowicz, D. Szewieczek, and L. A. Dobrzański, "New possibilities of application of composite materials with soft magnetic properties," J. Achiev. Mater. Manuf. Eng., vol. 20, 2007.

[24] Universidad Complutense Madrid "Laboratorio de Materiales Magnéticos, Ciclo de histéresis de materiales ferromagnéticos," $2016 . \quad$ [Online]; Available: https://www.ucm.es/data/cont/media/www/pa g-17833//Materiales magnéticos 2015-16.pdf

[25] S. Briceño et al., "Síntesis, caracterización y propiedades magnéticas de las nanopartículas de $\mathrm{CoFe} 2 \mathrm{O} 4$ usando PEG como surfactante," Rev. Latinoam. Metal. y Mater., vol. 33, no. 1, 2013.

[26] L. A. Ramajo, A. A. Cristóbal, P. M. Botta, J. M. Porto López, M. M. Reboredo, and M. S. Castro, "Dielectric and magnetic response of Fe304/epoxy composites," Compos. Part A Appl. Sci. Manuf., vol. 40, no. 4, pp. 388-393, Apr. 2009.

[27] R. Pearson, "Las llantas: un problema más de contaminación,” 2015. [Online]. Available: https://www.launion.com.mx/opinion/ecologia -en-tus-manos/noticias/79652-las-llantas-unproblema-más-de-contaminación.html.

[28] C. Guevara, "Cada día más de 2.050 llantas terminan invadiendo el espacio público," $E l$ Tiempo, 2015. [Online]. Available: https://www.eltiempo.com/archivo/documento /CMS-15317455.

[29] Universidad Politécnica de Valencia, "Curso de Fundamentos de Ciencia de Materiales." [Online]. Available: https://www.upv.es/materiales/Fcm/Fcm10/fc m10_5.html.

[30] L. M. Olaya-Guarín, "Problemática y reciclaje de llantas: experiencia exitosa en la problemática y reciclaje de llantas en el municipio de Apulo," Universidad Militar Nueva Granada 